\title{
Small-Scale Effect on Longitudinal Wave Propagation in Laser-Excited Plates
}

\author{
F. Kh. Mirzade \\ Institute on Laser and Information Technologies, Russian Academy of Sciences, Moscow 140700, Russia \\ Correspondence should be addressed to F. Kh. Mirzade; fmirzade@rambler.ru
}

Received 29 June 2014; Revised 5 October 2014; Accepted 6 October 2014; Published 21 October 2014

Academic Editor: Ana Benito

Copyright (C) 2014 F. Kh. Mirzade. This is an open access article distributed under the Creative Commons Attribution License, which permits unrestricted use, distribution, and reproduction in any medium, provided the original work is properly cited.

Longitudinal wave propagation in an elastic isotopic laser-excited solid plate with atomic defect (vacancies, interstitials) generation is studied by the nonlocal continuum model. The nonlocal differential constitutive equations of Eringen are used in the formulations. The coupled governing equations for the dynamic of elastic displacement and atomic defect concentration fields are obtained. The frequency equations for the symmetrical and antisymmetrical motions of the plate are found and discussed. Explicit expressions for different characteristics of waves like phase velocity and attenuation (amplification) coefficients are derived. It is shown that coupling between the displacement and defect concentration fields affects the wave dispersion characteristics in the nonlocal elasticity. The dispersion curves of the elastic-diffusion instability are investigated for different pump parameters and larger wave numbers.

\section{Introduction}

The studies on the interplay of strain field and defect concentration field are of importance in various branches of science and technology, particularly in the laser fast recrystallization, laser annealing, multipulse laser etching, and pulsed laser-assisted thin-film deposition. During the past years, several models for self-organization processes of ordered large-scale (strain-concentration) structures in an ensemble of interacting (through the strain field) atomic defects (interstitial atoms, vacancies) on the surface of the laserirradiated solid half-space [1,2] and in solid layers [3-6] have been considered using coupled evolution equations for the atomic defect concentration field and the classical (local) elasticity equations for the self-consistent displacement field of the medium. The formation of one-dimensional (1D) nonlinear localized deformational structures in metallic and semiconductor plates has been investigated taking into account the influence of temperature changes, geometrical dispersion due to the presence of the boundaries, and dispersions due to defect-elastic interaction and flexoelectricity [3]. Also, the conditions needed for clusters and periodic defect-deformation structures to emerge were found, and the characteristics of those structures-such as the period of periodic structure, and the spatial distributions of strain and defect concentration fields-were determined.

The classical continuum elasticity, which is a scale-free theory, cannot predict the small size effects. At nanometer scales, size effects become prominent. The classical elasticity concept is inadequate for describing the formation of small-scale ordered structures and mechanical (static or dynamic) behavior of microstructured materials, because their behavior is characterized by nonlocal stresses and the existence of an internal length scale [7, 8]. Therefore, development of appropriate nonlocal elasticity mathematical models for nanostructure formation and nanomaterials is of great importance.

The studies of small-scale effects by using the NLE theory have been an area of active research. Various nonlocal theories of linear elasticity have been proposed to describe the scale effects on the characteristics of the vibration and elastic wave propagation in the above-mentioned submicrosized or nanosized structures. The basic constitutive equations and the governing equations of linear NLE theory were derived by Eringen [7, 8] and Kunin [9]. Later the nonlocal theories have been applied for the analysis of micro- and nanoscale plate-like structures, in which the small-scale effects become significant. In [10] Eringen considered vibration behavior of 
a nanoplate by using linear theory of nonlocal continuum mechanics. By using [7], Reddy [11] reformulated the classical plate theories. The nonlocal scale influences on the wave dispersion properties of the nanoplates and nanorods are discussed in detail in [12-14]. Vibration characteristics of nanoplates, based on three-dimensional theory of elasticity employing nonlocal continuum mechanics, have been discussed in [15]. A review of some other applications of nonlocal elasticity theories for nanostructures can be found in [16]. Propagation of longitudinal elastic and thermoelastic waves in isotropic, homogeneous infinite medium and plates with long-rang interactions has been studied by Nowiński $[17,18]$. The NLE theory for the propagation of surface waves in an isotropic laser-excited elastic solid half-space with mobile atomic point defects has been proposed in [19]. A qualitative validation study showed that results based on the nonlocal continuum mechanics are in agreement with the experimental reports in this field.

In this present investigation, we propose examining the propagation of longitudinal plane waves in laser-excited solid plates with atomic defect generation by using nonlocal continuum model. The surfaces of the plates are assumed to be free of stresses. Secular equation that governs the propagation of small-amplitude elastic-concentration waves has been derived by solving a system of coupled linearized nonlocal field equations. We obtain the frequency equations for symmetric and antisymmetric motions about the plane of symmetry of the plate. The solution of the frequency equations is obtained and discussed, by assuming that the coupling parameter is small. It is found that the influence of coupling between the displacement and defect-concentration fields affects the phase velocity and attenuation (amplification) constant in the NLE theory. It is noticed that, at short wavelength limits, the motion for longitudinal waves is governed by Rayleigh-Lamb-type secular equation.

\section{Basic Governing Equations}

We consider a linear, isotropic elastic solid with nonlocal properties where mobile atomic point defects are excited by an external energy flux (e.g., laser radiation). Let $n\left(x_{i}, t\right)$, $i=1,2,3$ be the concentration of these defects at a point $x_{i}$ at a time $t$. There could be two types of defect but we limit our consideration to one. The constitutive relations in such solid, involving atomic defect generation, can be written by an equivalent differential form as follows [19]:

$$
\begin{gathered}
\sigma_{i k}(\mathbf{x})=\left(1+g^{2} \nabla^{2}\right) s_{i k}(\mathbf{x})-\left(1+h^{2} \nabla^{2}\right) \beta_{0} \delta_{i k} n(\mathbf{x}), \\
s_{i k}(\mathbf{x}, t)=\lambda_{0} e(\mathbf{x}, t) \delta_{i k}+2 \mu_{0} e_{i k}(\mathbf{x}, t), \quad i, k=1,2,3 .
\end{gathered}
$$

Here $\sigma_{i k}$ is the nonlocal stress tensor and $h$ and $g$ are the intrinsic characteristic length scale parameters, characterizing the defect-atom and atom-atom interactions, respectively; $2 g^{2}=\int \alpha(|\tau|) \tau^{2} d \tau / \int \alpha(|\tau|) d \tau ; 2 h^{2}=\int \beta(|\tau|) \tau^{2} d \tau /$ $\int \beta(|\tau|) d \tau ; \beta_{0}=\int \beta(|\tau|) d \tau=K \Omega_{d}$ is deformation potential of the defect ( $\Omega_{d}$ is the change of the volume of the medium under formation of a single defect and $K$ is the bulk modulus). The functions $\alpha$ and $\beta$ are known as atom-atom (short-range) and atom-defect (long-range) interaction kernels or moduli of nonlocality, which decay smoothly with distance. Nonlocal moduli are physical properties of materials like other physical constants and need to be determined experimentally. Various different forms of $\alpha$ and $\beta$ may be found in $[7,20]$.

Equation (2) is the classical constitutive relation where $s_{i k}(\mathbf{x}, t)$ is the classical stress tensor at point $(\mathbf{x})$ in the body, at time $t$, which is related to the linear strain tensor $e_{i k}(\mathbf{x}, t)$ at the same point; $\lambda_{0}$ and $\mu_{0}$ are the Lame's constants; $e_{i k}$ is the strain tensor; $e=u_{k, k}$ is the dilatation (a subscript preceded by comma denotes differentiation with respect to the corresponding coordinate: $\left.(\cdot)_{, i}=\partial(\cdot) / \partial x_{i}\right) ; e_{i k}(\mathbf{x}, t)=$ $\left(u_{i, k}(\mathbf{x}, t)+u_{k, i}(\mathbf{x}, t)\right) / 2$ is the classical strain tensor $\left(u_{i}\right.$ are the components of displacement vector $(\mathbf{u}))$; and $\nabla^{2}$ is the Laplacian operator.

The scale coefficients $g$ and $h$ in (1) are dependent on the lattice or atomic chain model used and the interatomic potentials assumed. The values of these material parameters are taken to be order of interatomic distance. Thus, the scale coefficients $g$ and $h$ in the modeling will lead to small-scale effect on the response of structures in nanosize. In the limit when the effect of strains at points other than $\mathbf{x}$ is neglected, one obtains classical (local) model of elasticity by setting $g=$ $h=0$.

It should be pointed out that (1) has the same structure as the constitutive relation for second order gradient elasticity in solid mechanics (without the $n$ term) [21] or gradient thermoelasticity, with $n$ playing the role of temperature [7]. Here the stress tensor depends not only on the components of the strain tensor and the defect concentration but also on their derivatives.

In the context of the NLE theory, the basic field equations for the displacement vector and the defect concentration for a nonlocal solid neglecting the body forces can be written as [19]

$$
\begin{aligned}
\rho \frac{\partial^{2} \mathbf{u}}{\partial t^{2}}= & \left(1+g \nabla^{2}\right)\left[\mu_{0} \nabla^{2} \mathbf{u}+\left(\lambda_{0}+2 \mu_{0}\right) \nabla(\nabla \cdot \mathbf{u})\right] \\
& -\left(1+h \nabla^{2}\right) \beta_{0} \nabla n \\
\frac{\partial n}{\partial t} & =G+D \nabla^{2} n-\frac{D}{k_{B} T} \nabla(n \mathbf{f})-n \tau^{-1} .
\end{aligned}
$$

In these equations $\rho$ is the density of the medium; $G=$ $G_{0} \exp \left(-w_{g} / k_{B} T\right)$ is the thermal-fluctuation generation rate of atomic defects at sites $\left(G_{0}\right.$ is the constant and $k_{B}$ is the Boltzmann constant); $\tau=\tau_{0} \exp \left(w_{m} / k_{B} T\right)$ is the relaxation time of defects; $w_{g}$ and $w_{m}$ are the formation and migration energies for the defects in crystals. It is assumed that the generation rate $(G)$ is spatially uniform.

The first term in the right-hand side of (4) takes into account laser-induced generation of defects, the second term represents diffusion with a coefficient $D$, the third term corresponds to the drift of defects under the influence of the force $\mathbf{f}=-\nabla U_{\text {int }}$ resulting from the nonlocal interaction of defects with an inhomogeneous strain field, and the fourth term describes the rate of their disappearance due to recombination processes. The interaction energy $U_{\text {int }}$ of a 
defect with the strain field $(e)$ in a nonlocal solid is given by [19]: $U_{\text {int }}=-\beta_{0}\left(e+h^{2} \nabla^{2} e\right)$.

\section{Nonlocal Equations and Boundary Conditions}

We consider an elastic plate of thickness $2 \mathrm{H}$ which extends to infinity in all directions. We assume that the middle plane of the plate coincides with the planes $x_{1}, x_{3}$ of the rectangular Cartesian coordinate system $0 x_{1} x_{2} x_{3}$. The upper and lower surfaces of the plate $\left(x_{2}= \pm H\right)$ are supposed to be free of stresses. We consider the longitudinal waves propagating along the $x_{1}$-direction in plates so that all particles on a line parallel to $x_{3}$ axis are equally displaced. Therefore, the displacement component $u_{3}$ in the $x_{3}$-direction in everywhere zero $\left(u_{3}=0\right)$, and all the field quantities are taken to be independent of $x_{3}$-coordinate. Denoting $u_{1}$ and $u_{2}$ as the nonzero components of the displacement vector $(\mathbf{u})$, we set $u_{1}=$ $u_{1}\left(x_{1}, x_{2}, t\right)$ and $u_{2}=u_{2}\left(x_{1}, x_{2}, t\right)$ and consequently write for the strain tensor $\left(e_{i j}\right): e_{11}=u_{1,1}, e_{22}=u_{2,2}, 2 e_{12}=u_{1,2}+u_{2,1}$.

Elaborating (1) the two-dimensional nonlocal constitutive equations will be

$$
\begin{aligned}
\sigma_{11}= & \left(1+g^{2} \nabla^{2}\right)\left[\left(\lambda_{0}+2 \mu_{0}\right) u_{1,1}+\lambda_{0} u_{2,2}\right] \\
& -\left(1+h^{2} \nabla^{2}\right) \beta_{0} n, \\
\sigma_{22}= & \left(1+g^{2} \nabla^{2}\right)\left[\left(\lambda_{0}+2 \mu_{0}\right) u_{2,2}+\lambda_{0} u_{1,1}\right] \\
& -\left(1+h^{2} \nabla^{2}\right) \beta_{0} n, \\
\sigma_{12}= & \left(1+g^{2} \nabla^{2}\right) \mu_{0}\left(u_{1,2}+u_{2,1}\right),
\end{aligned}
$$

where $\nabla^{2}=\partial^{2} / \partial x_{1}^{2}+\partial^{2} / \partial x_{2}^{2}$.

Then the equations of motion governing the in-plane vibration of the plates are written as

$$
\begin{aligned}
& \rho \frac{\partial^{2} u_{1}}{\partial t^{2}}=\sigma_{11,1}+\sigma_{12,2}, \\
& \rho \frac{\partial^{2} u_{2}}{\partial t^{2}}=\sigma_{21,1}+\sigma_{22,2} .
\end{aligned}
$$

Combining now (5a), (5b), and (5c) with (6) leads to the following system of coupled partial differential equations for the displacement components $u_{1}$ and $u_{2}$ :

$$
\begin{aligned}
\rho \frac{\partial^{2} u_{1}}{\partial t^{2}}= & \left(1+g^{2} \nabla^{2}\right) \\
& \times\left[\left(\lambda_{0}+2 \mu_{0}\right) u_{1,11}+\mu_{0} u_{1,22}+\left(\lambda_{0}+\mu_{0}\right) u_{2,12}\right] \\
& -\beta_{0}\left(1+h^{2} \nabla^{2}\right) n_{, 1}, \\
\rho \frac{\partial^{2} u_{2}}{\partial t^{2}}= & \left(1+g^{2} \nabla^{2}\right) \\
& \times\left[\mu_{0} u_{2,11}+\left(\lambda_{0}+2 \mu_{0}\right) u_{2,22}+\left(\lambda_{0}+\mu_{0}\right) u_{1,12}\right] \\
& -\beta_{0}\left(1+h^{2} \nabla^{2}\right) n_{, 2} .
\end{aligned}
$$

We can express the defect concentration field as $n=n_{0}+N$ ( $n_{0}=G \tau$ is a spatially homogeneous solution; $N$ is a small nonhomogeneous perturbations due to elastic wave propagation). Substituting this expression into (4) and neglecting the nonlinear terms, we get the linearized equation as follows:

$$
\begin{aligned}
& \left(\frac{\partial}{\partial t}+\frac{1}{\tau}\right) N-D\left(N_{, 11}+N_{, 22}\right) \\
& =-\frac{n_{0} \beta_{0} D}{k_{B} T}\left(1+g^{2} \nabla^{2}\right)\left(e_{, 11}+e_{, 22}\right),
\end{aligned}
$$

where $l=\sqrt{D \tau}$.

The boundary conditions at the free surfaces are the vanishing of normal stress, tangential stress, and defect-concentration gradient; that is,

$$
\sigma_{22}=0, \quad \sigma_{21}=0, \quad n_{, 2}=0
$$

on planes $x_{2}= \pm H$.

The system of (7)-(8) is closely coupled. $\partial^{2} \vec{u} / \partial t^{2}$ in (7) depends on the defect concentration field $(N)$ and $\partial N / \partial t$ in (8) depends on elastic displacement field (u).

We express the displacement components $u_{1}$ and $u_{2}$ in terms of Lame-type potentials $\varphi$ and $\psi$ as

$$
u_{1}=\varphi_{, 1}+\psi_{, 2}, \quad u_{2}=\varphi_{, 2}-\psi_{, 1} \text {, }
$$

where $\psi$ and $\varphi$ depend only $x_{1}, x_{2}$, and $t$. Now (7)-(8) can be written as

$$
\begin{gathered}
\rho \frac{\partial^{2} \psi}{\partial t^{2}}=\mu_{0}\left(1+g^{2} \nabla^{2}\right) \nabla^{2} \psi \\
\rho \frac{\partial^{2} \varphi}{\partial t^{2}}=\left(\lambda_{0}+2 \mu_{0}\right)\left(1+g^{2} \nabla^{2}\right) \nabla^{2} \varphi-\beta_{0}\left(1+h^{2} \nabla^{2}\right) N \\
\left(\frac{\partial}{\partial t}+\frac{1}{\tau}\right) N-D\left(N_{, 11}+N_{, 22}\right)=-\frac{n_{0} \beta_{0} D}{k_{B} T}\left(1+h^{2} \nabla^{2}\right) \nabla^{4} \varphi
\end{gathered}
$$

Equation (11) is uncoupled, whereas (12) and (13) are coupled in $\varphi$ and $N$. From (12) and (13), we see that while the longitudinal wave is affected due to the presence of defect concentration fields, the transverse wave remains unaffected. The solution of (11) corresponds to purely transverse waves in the solid.

Hence, the mathematical formulation of the problem is to solve (11)-(13) under the above boundary conditions.

\section{Method of Solution}

To solve (11)-(13), we assume that the variation of all variables with $x_{1}$ and $t$ is of the form $\exp \left[i\left(k x_{1}+\omega t\right)\right]$ and we take $\varphi$, $\psi$, and $N$ in the forms

$$
[\varphi, \psi, N]=\left[\widetilde{\varphi}\left(x_{2}\right), \widetilde{\psi}\left(x_{2}\right), \widetilde{N}\left(x_{2}\right)\right] \exp \left[i\left(k x_{1}+\omega t\right)\right],
$$


where $\omega$ is the frequency of wave propagation, $k$ is the wave number ( $k=2 \pi / \Lambda, \Lambda$ is the wave-length); the phase velocity is given by $c=\omega_{r} / k$ and attenuation constant by $P=-\omega_{i}$, where $\omega_{r}=\operatorname{Re}(\omega)$ and $\omega_{i}=\operatorname{Im}(\omega)$ mean, respectively, the real and imaginary parts of $\omega$.

Now (11)-(13) reduce to

$$
\begin{gathered}
\widetilde{\varphi}_{, 22}-\left(k^{2}-\rho \frac{\omega^{2}}{\lambda+2 \mu}\right) \widetilde{\varphi}-\frac{\beta}{\lambda+2 \mu} \widetilde{N}=0 \\
\widetilde{\psi}_{, 22}-\left(k^{2}-\rho \frac{\omega^{2}}{\mu}\right) \widetilde{\psi}=0, \\
\widetilde{N}_{, 22}-\left(k^{2}+\frac{i \omega \tau+1}{l^{2}}\right) \widetilde{N}-\frac{n_{0} \beta}{k_{B} T}\left(\widetilde{\varphi}_{, 2222}-2 k^{2} \widetilde{\varphi}_{, 22}+k^{4} \widetilde{\varphi}\right),
\end{gathered}
$$

where $\lambda=\lambda(k)=\lambda_{0}\left(1-g^{2} k^{2}\right)$ and $\mu=\mu(k)=\mu_{0}(1$ $\left.-g^{2} k^{2}\right)$ are the nonlocal elastic moduli; $\beta=\beta(k)=\beta_{0}(1$ $\left.-h^{2} k^{2}\right)$ is the nonlocal constant characterizing lattice deformation due to atomic defects; $\widetilde{\varphi}\left(k, x_{2}\right), \widetilde{\psi}\left(k, x_{2}\right)$, and $\widetilde{N}\left(k, x_{2}\right)$ are unknown functions (amplitude functions); and $l=$ $\sqrt{D \tau}$. In derivation of (15)-(17), it is assumed that the longrange internal influences of particles rather rapidly with increasing distance from the particle and the functions that characterize the particle interactions in the $x_{2}$-direction can be approximated in terms of delta-like functions.

Eliminating $\widetilde{N}$ from (15) and (17), we obtain

$$
\begin{gathered}
(1-\delta) \widetilde{\varphi}_{, 2222}-\left[2 k^{2}(1-\delta)-\frac{\rho \omega^{2}}{\lambda+2 \mu}+\frac{i \omega \tau+1}{l^{2}}\right] \widetilde{\varphi}_{, 22} \\
+\left[\left(k^{2}-\frac{\rho \omega^{2}}{\lambda+2 \mu}\right)\left(k^{2}+\frac{i \omega \tau+1}{l^{2}}\right)-\delta k^{4}\right] \tilde{\varphi}=0,
\end{gathered}
$$

where $\delta=n_{0} \beta^{2} /(\lambda+2 \mu) k_{B} T$ is the coupling constant of nonlocal defect-strain interaction.

The solutions of (16) and (18) are given as

$$
\begin{gathered}
\widetilde{\varphi}\left(x_{2}\right)=\sum_{j=1}^{2}\left(a_{j} \cosh \eta_{j} x_{2}+b_{j} \sinh \eta_{j} x_{2}\right), \\
\widetilde{\psi}\left(x_{2}\right)=a_{3} \sinh \eta_{3} x_{2}+b_{3} \cosh \eta_{3} x_{2},
\end{gathered}
$$

where $a_{j}, b_{j}, j=1,2,3$ are the arbitrary constants and

$$
\begin{gathered}
\eta_{3}^{2}=k^{2}-\frac{\rho \omega^{2}}{\mu}, \\
\eta_{1}^{2}+\eta_{2}^{2}=\left[2 k^{2}(1-\delta)-\rho \frac{\omega^{2}}{\lambda+2 \mu}+\frac{i \omega \tau+1}{l^{2}}\right](1-\delta)^{-1}, \\
\eta_{1}^{2} \eta_{2}^{2}=\left[\left(k^{2}-\rho \frac{\omega^{2}}{\lambda+2 \mu}\right)\left(k^{2}+\frac{i \omega \tau+1}{l^{2}}\right)-\delta k^{4}\right](1-\delta)^{-1} .
\end{gathered}
$$

From (20a) we see that the root $\eta_{3}^{2}$ corresponds to the transverse wave, whereas the roots $\eta_{1}^{2}$ and $\eta_{2}^{2}$ correspond to the coupled longitudinal elastic and defect concentration waves.

To find explicit expressions for $\eta_{1}^{2}$ and $\eta_{2}^{2}$ we seek solutions of (20a), (20b), and (20c) for small values of the coupling constant between the field of defect concentration and the field of strain. Since coupling coefficient is a physical characteristic of the medium, so the effect of damping and dispersion of elastoconcentration waves depends exclusively on the values of this coefficient. Assuming that the coupling constant is sufficiently small, $(\delta \ll 1)$, after the first order approximation in $\delta$, for the roots $\eta_{1}^{2}$ and $\eta_{2}^{2}$, we have

$$
\eta_{1}^{2}=\bar{\eta}_{1}^{2}+\delta \tilde{\eta}_{1}^{2}+O\left(\delta^{2}\right), \quad \eta_{2}^{2}=\bar{\eta}_{2}^{2}+\delta \tilde{\eta}_{2}^{2}+O\left(\delta^{2}\right),
$$

where

$$
\begin{gathered}
\bar{\eta}_{1}^{2}=k^{2}-\frac{\rho \omega^{2}}{(\lambda+2 \mu)}, \quad \bar{\eta}_{2}^{2}=k^{2}+\frac{(i \omega \tau+1)}{l^{2}}, \\
\widetilde{\eta}_{1}^{2}=-\left(\bar{\eta}_{1}^{2}-k^{2}\right)^{2} \Theta^{-1}(\omega), \quad \tilde{\eta}_{1}^{2}=\left(\bar{\eta}_{2}^{2}-k^{2}\right)^{2} \Theta^{-1}(\omega), \\
\Theta(\omega)=\frac{\rho \omega^{2}}{(\lambda+2 \mu)}+(i \omega \tau+1) l^{-2} .
\end{gathered}
$$

\section{Derivation of the Dispersion Equations}

Using the boundary conditions (9), one obtains a system of six algebraic equations for determination of the constants $a_{j}$ and $b_{j}$. This system has a nontrivial solution if the determinant of their coefficients vanishes. This leads to

$$
\left|L_{i k}\right| \times\left|S_{i k}\right|=0, \quad i, k=1,2,3,
$$

where

$$
\begin{gathered}
L_{1 j}=\left(2-\frac{\rho \omega^{2}}{\mu k^{2}}\right) \operatorname{coth} \eta_{j} H, \\
L_{13}=2 i \eta_{3} k^{-1} \operatorname{coth} \eta_{3} H, \\
L_{2 j}=2 i \eta_{j} k^{-1}, \\
L_{23}=-\left(2-\frac{\rho \omega^{2}}{k^{2} \mu}\right), \\
L_{3 j}=\eta_{j} k^{-1}\left(\eta_{j}^{2}+\frac{\rho \omega^{2}}{\mu}-k^{2}\right), \\
L_{33}=0, \\
j=1,2 .
\end{gathered}
$$

Expressions for $S_{i k}$ 's can be obtained from $L_{i k}$ by replacing $\operatorname{coth}\left(\eta_{j} H\right)$ by $\tanh \left(\eta_{j} H\right)$. Equations $\left|L_{i k}\right|=0$ and $\left|S_{i k}\right|=0$, respectively, are associated with the symmetric and antisymmetric motions of the plate relative to the middle surface. The 
frequency equations $\left|S_{i k}\right|=0$ and $\left|A_{i k}\right|=0$ can be combined and represented as

$$
\begin{aligned}
k^{2} \eta_{1} \eta_{2} \eta_{3}\left(\eta_{2}^{2}-\eta_{1}^{2}\right)= & \left(k^{2}-\rho \frac{\omega^{2}}{2 \mu}\right)^{2} \\
& \times\left[\frac{\eta_{2}\left(\eta_{2}^{2}-\bar{\eta}_{1}^{2}\right)}{Z_{1}^{\mp}}-\frac{\eta_{1}\left(\eta_{1}^{2}-\bar{\eta}_{1}^{2}\right)}{Z_{2}^{\mp}}\right]
\end{aligned}
$$

$\left(Z_{i}=\tanh \eta_{i} H / \tanh \eta_{3} H, i=1,2\right)$. Here the superscript +1 corresponds to antisymmetric and -1 refers to symmetric modes.

Equations (25) are the phase velocity equations for the waves in nonlocal solid plate, where $\eta_{1}, \eta_{2}$, and $\eta_{3}$ are given by (20a), (20b), and (20c). They show dispersive character of wave propagation in plates. Dispersive nature of the general waveform arises due to defect generation and nonlocal character of atom-defect and atom-atom interactions in the medium.

From (25) and the corresponding equation governing the dispersion of strain-diffusive waves in plates without scale effects from [22], we readily see that the form of two equations is the same, but the expressions for $\eta_{1}, \eta_{2}, \eta_{3}$, and $\mu$ entering in them are not one and the same.

Equations (25) may be regarded as the revised forms of the classical local equations obtained by Raleigh and Lamb in an elastic layer with defect generation and small-scale length effects. The assumption of vanishing defect generation in these equations gives us exactly the same results as those obtained by Nowiński [17] for thin plates with long-range interactions; that is,

$$
\left(\frac{\tanh \eta_{3} H}{\tanh \bar{\eta}_{1} H}\right)^{ \pm 1}-\frac{\left(\eta_{3}^{2}+k^{2}\right)^{2}}{4 q^{2} \bar{\eta}_{1} \eta_{3}}=0 .
$$

These resulting dispersion relations have already been discussed in detail by Achenbach for various possibilities and situations in elastokinetics [23].

\section{Solution of Dispersion Equation}

In general, the obtained dispersion equations are complex and a general discussion of their solutions presents considerable difficulties. This equation can be solved numerically or graphically, but in some cases of interest it can also be solved analytically.

In general case (25) has solutions describing qualitatively different types of instability: (1) instability of frequencies of surface acoustic waves and (2) generation of ordered surface (static) diffusion-strain (DS) nanosized structures.

6.1. Instability of Frequencies of Surface Waves. The assumption $\delta \ll 1$ makes it possible to expand the following quantities in power series of $\delta$ :

$$
\begin{gathered}
\eta_{2}^{2}-\eta_{1}^{2}=\Theta+\delta\left(\widetilde{\eta}_{2}^{2}-\widetilde{\eta}_{1}^{2}\right), \quad \eta_{1}^{2}-\bar{\eta}_{1}^{2}=\delta \widetilde{\eta}_{1}^{2}, \\
\eta_{2}^{2}-\bar{\eta}_{1}^{2}=\Theta+\delta \widetilde{\eta}_{2}^{2}, \quad \tanh \eta_{1} H=\tanh \bar{\eta}_{1} H+\delta Y,
\end{gathered}
$$

$$
\eta_{1}=\bar{\eta}_{1}+\frac{\delta \widetilde{\eta}_{1}^{2}}{2 \bar{\eta}_{1}}, \quad \eta_{2}=\bar{\eta}_{2}+\frac{\delta \widetilde{\eta}_{2}^{2}}{2 \bar{\eta}_{2}}
$$

where $Y=2 \bar{\eta}_{1}^{-1} \widetilde{\eta}_{1}^{2} H \exp 2 \bar{\eta}_{1} H\left(1+\exp 2 \bar{\eta}_{1} H\right)^{-2}$.

Then (25) for the symmetric modes transforms to

$$
R(\omega, k)=\delta L(\omega, k, \delta),
$$

where

$$
\begin{gathered}
R(\omega, k)=\frac{\left(\eta_{3}^{2}+k^{2}\right)^{2}}{4 q^{2} \bar{\eta}_{1} \eta_{3}} \frac{\tanh \eta_{3} H}{\tanh \bar{\eta}_{1} H}-1, \\
L(\omega, k, \delta)=\delta\left(\frac{\widetilde{\eta}_{1}^{2}}{2 \bar{\eta}_{1}^{2}}-\frac{\widetilde{\eta}_{1}^{2}}{\Theta}+\frac{Y}{\tan \bar{\eta}_{1} H}+\frac{\bar{\eta}_{1} \widetilde{\eta}_{1}^{2}}{\Theta \bar{\eta}_{2}} \frac{\tanh \bar{\eta}_{1} H}{\tanh \bar{\eta}_{2} H}\right) \\
=\delta L_{0}(\omega, k) .
\end{gathered}
$$

If the defect concentration and strain fields are not coupled with each other, then the coupling constant $\delta$ is identically zero $(\delta \equiv 0)$. In this case from (28) we obtain the nonlocal elastic solution given by Mirzade [19]. In general case $(\delta \neq$ 0 ), introducing the dimensionless variable $\xi=\left(\omega / c_{T} k\right)^{2},(28)$ becomes

$$
R(\xi)=L(\xi, \delta)
$$

Now we may consider that $\varepsilon$ is the increment of the value $\xi$ due to $\delta \neq 0$. Then (30) can be written in the form of

$$
R\left(\xi_{R}+\varepsilon\right)=L\left(\xi_{R}+\varepsilon, \delta\right)
$$

$\left(\xi_{R}\right.$ is a root of the equation $\left.R\left(\xi_{R}\right)=0\right)$.

Assuming that $\varepsilon \ll 1$, we may expand both sides of (31) into a Taylor series in the vicinity of the point $\xi=\xi_{R}$. Retaining only the first two terms, we obtain

$$
\begin{gathered}
R\left(\xi_{R}\right)+\varepsilon A\left(\xi_{R}\right)=L\left(\xi_{R}, \delta\right)+\varepsilon B\left(\xi_{R}, \delta\right), \\
\varepsilon\left(\xi_{R}, \delta\right)=\frac{L\left(\xi_{R}, \delta\right)}{A\left(\xi_{R}\right)-B\left(\xi_{R}, \delta\right)} \approx \frac{L_{0}\left(\xi_{R}\right)}{A\left(\xi_{R}\right)} \delta=\varepsilon_{r}+i \varepsilon_{i},
\end{gathered}
$$

where $A=\left.(\partial R / \partial \xi)\right|_{\xi=\xi_{R}}, B=\left.(\partial L / \partial \xi)\right|_{\xi=\xi_{R}}, \varepsilon_{r}=\operatorname{Re}(\varepsilon)$, and $\varepsilon_{i}=\operatorname{Im}(\varepsilon)$.

The real part of $\varepsilon$ characterizes the change of the phase velocity and its imaginary part $\left(\varepsilon_{i}\right)$ defines the attenuation constant.

Since $\xi=\left(\omega / c_{T} k\right)^{2}=\xi_{R}+\varepsilon$, we have $\omega=$ $q c_{T}\left(\xi_{R}+\varepsilon_{r}+i \varepsilon_{i}\right)^{1 / 2}$. This equation gives the following expressions:

$$
\omega_{r}=c_{T} k \sqrt{\xi_{R}}\left(1-\delta \frac{\left|L_{0 r}\right|}{2 \xi_{R} A}\right), \quad \omega_{i}=c_{T} k \delta \frac{L_{0 i}}{\xi_{R} A},
$$

where $L_{0 r}=\operatorname{Re}\left(L_{0}\right), L_{0 i}=\operatorname{Im}\left(L_{0}\right)$. 
Then, for the phase velocity and attenuation constant of surface waves, we have

$$
\begin{gathered}
c=c_{T} \sqrt{\xi_{R}}\left(1-\delta \frac{\left|L_{0 r}\right|}{2 \xi_{R} A}\right), \\
P=-c_{T} k \delta \frac{L_{0 i}}{\xi_{R} A} .
\end{gathered}
$$

Since $\delta>0$, in this case there is a softening of frequencies of acoustic waves (instability of frequencies $\omega_{r} \rightarrow 0$ and $\omega_{i}>$ 0 ) and this is related to taking into account the generation of atomic defects. It is necessary to notice that reduction of frequency occurs not up to zero, and up to value $\omega_{\eta}=$ $\eta_{v} k^{2} / 2 \rho \ll c_{L} k$ ( $\eta_{v}$ is the viscosity coefficient). Equation (35b) describes attenuation of the amplitudes of acoustic waves.

In the following we will consider two limited cases.

(i) Long waves. For long waves one has to consider the limits at $k H \rightarrow 0$. Calculations give us that the local and nonlocal dispersion expressions of the long wave limit become identical as it is expected.

(ii) Short waves. For short waves when the wavelength with respect to the thickness of the plate is quite small, so that $\eta_{i} H \gg 1$. In this approximation the wave number becomes large $(k H \rightarrow \infty)$ and we may assume that $\tanh \eta_{i} H \approx 1$. Then expressions for $B$ and $A$ in (33) become

$$
\begin{aligned}
\left.\left(\frac{\partial R}{\partial \xi}\right)\right|_{\xi=\xi_{R}} & =B \\
& =\frac{\zeta}{2\left(1-\zeta \xi_{R}\right)}+\frac{\zeta}{2\left(1-\xi_{R}\right)}-\frac{1}{\left(1-0.5 \xi_{R}\right)}
\end{aligned}
$$

$A\left(\xi_{R}\right)$

$$
\begin{gathered}
=-\left(\zeta \xi_{R}\right)^{2}\left(2 \sqrt{1-\zeta \xi_{R}}+\sqrt{1+\frac{\left(i k c_{T} \tau \sqrt{\xi_{R}}+1\right)}{(l k)^{2}}}\right) \\
\quad \times\left(2\left(1-\zeta \xi_{R}\right) \sqrt{1+\frac{\left(i k c_{T} \tau \sqrt{\xi_{R}}+1\right)}{(l k)^{2}}}\right. \\
\left.\quad \times\left(\sqrt{1-\zeta \xi_{R}}+\sqrt{1+\frac{\left(i k c_{T} \tau \sqrt{\xi_{R}}+1\right)}{(l k)^{2}}}\right)^{2}\right)^{-1}
\end{gathered}
$$

In this case, expressions (34) for the frequencies transform to results for the Rayleigh surface waves in elastic semispace solids with atomic defect generation which were obtained and discussed in detail, in [19, 22, 24]. Therefore, the phase velocity of waves in the plate tends to the velocity of Rayleigh surface waves.
The Rayleigh results enter here since, for such small wavelengths, the finite thickness plate appears as a halfspace. Hence, vibrational energy is transmitted mainly along the layer surface. Here, the dispersive character of wave propagation arises due to nonlocal character of atom-defect and atom-atom interactions in the medium and does not depend on thickness of plate.

6.2. Generation of Diffusion-Strain Nanosized Structures. Expanding $\eta_{1}$ and $\eta_{3}$ in powers of the small parameter $\omega_{i} / k c_{T \cdot L} \ll 1\left(\omega_{r}=0\right)$ and introducing the dimensionless wave number $k_{0}=\mathrm{kg}$, the life-time of defects $\tau_{0}=\tau \mathrm{D} / \mathrm{g}^{2}$, and parameters $\phi=\delta /(1-\zeta), \gamma=g^{2} / h^{2}$, we obtain from (25) the expression for the growth rate $\Gamma_{0}=\Gamma g^{2} / D\left(\Gamma=-\omega_{i}\right)$ of the diffusion-elastic instability [1] in the form

$$
\Gamma_{0}=\frac{k_{0}^{2}}{1-k_{0}^{2}}\left(k_{0}^{2}+\phi\left(1-\gamma k_{0}^{2}\right)^{2}-1\right)-\tau_{0}^{-1},
$$

where $\zeta=c_{T 0}^{2} c_{L 0}^{-2}\left(c_{L 0}\right.$ and $c_{T 0}$ are the classical longitudinal and the shear wave speeds in plates, resp.).

The dispersion equation (37) has two maximums. Figure 1 shows the dependences $\Gamma_{0}\left(\Lambda_{0}\right)$ of the increment on the wave length for two values of the control parameter $\phi=5$ and 15 when the ratio parameter is $\gamma=8$. The following typical values of parameters were used in the calculations: $h=0.6 \mathrm{~nm}, \phi=10$, and $D=4 \times 10^{-11} \mathrm{~cm}^{2} \mathrm{~s}^{-1}$. It follows from Figure 1(a) that the growth rate has a single maximum in the long-wave range (at $\Lambda_{m}=5 \times 10^{-6} \mathrm{~cm}$ ), at concentrations of defects that exceed the threshold value. However, at sufficiently high defect concentrations exceeding the threshold an additional maximum in the short-wavelength range appears (at $\Lambda<2 \times 10^{-6} \mathrm{~cm}$ ) (Figure 1(b)). Therefore, two gratings have the maximum growth rates.

Thus, a mechanism on the development of the diffusionelastic instability is because of the coupling between defect concentration dynamics and the elastic field of the plates.

Due to the nonlocal character of the defect-strain interactions the curve of the instability growth rate versus wave length has two maxima at sufficiently far above the instability threshold. This corresponds to the two scales of the surface relief modulation upon the laser irradiation of semiconductor layers. In the case of the laser molecular-beam epitaxy (MBE) and the laser-controlled deposition process the extrema (minima or maxima) of the surface relief formed by the DS structure serve as the nanoparticle nucleation and growth centers. Similarly, in the processes of multipulse laser etching, the rapid material removal also takes place on the extremes of the DS field, where the removal rate takes maximum values. As a result of this, on the surface of substrate, nanoparticle size distribution functions having two maxima can be formed. Transformation of the unimodal distribution to the bimodal distribution upon a variation in the irradiation regime is typical of the both $\mathrm{MBE}$ and the laser-induced surface nanostructuring of metals and semiconductors. Bimodal size distributions during the production of metal (gold) nanoparticles by pulsed laser deposition on substrates were detected experimentally in [25]. 


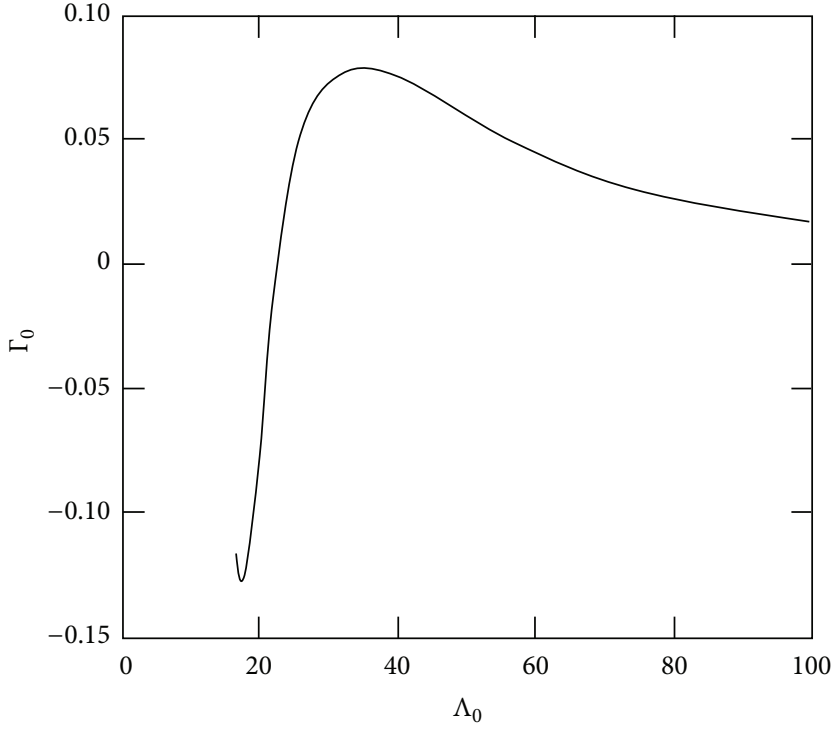

(a)

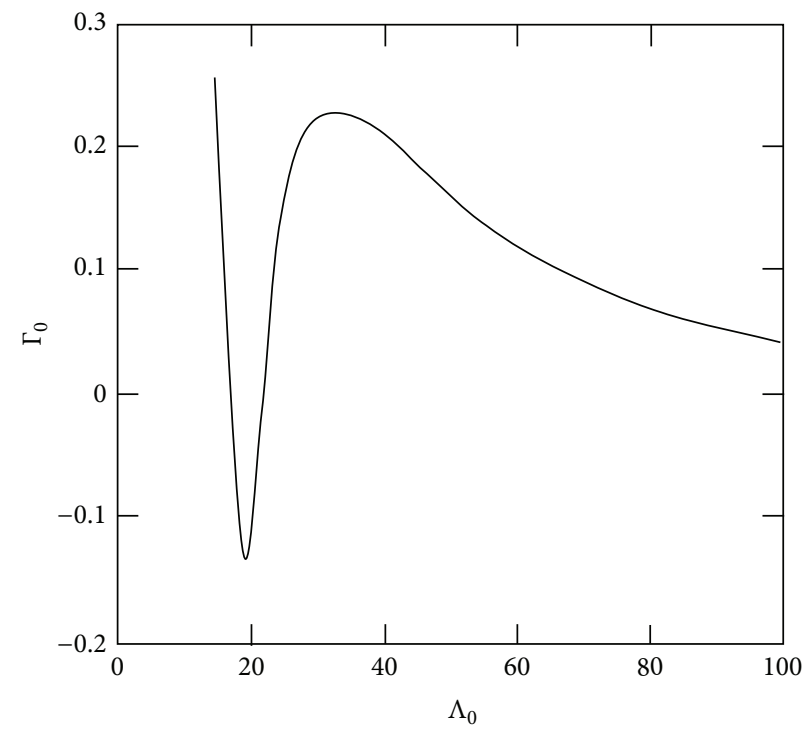

(b)

FIGURE 1: Dependences of the growth rate on the wavelength for two values of the control parameter $\phi: 5$ (a) and 15 (b) and ratio parameter: $\gamma=8$.

\section{Conclusions}

In this study, nonlocal continuum model for longitudinal wave propagation in an isotropic elastic plate containing a distribution of laser-induced atomic point defects has been presented based on Eringen's NLE theory. This theory assumes that the stress at a given point to be a functional of the strain field at every point in the solid. It allowed us to consider the small-scale effects that become significant when dealing with micro- and nanostructure formation. This theory has been extended to obtain the nonlocal second order strain gradient models of laser-excited plates with defect generation. Our analysis was based on the coupled nonlocal partial differential equations that consisted of the equations of elasticity taking into account the gradient of diffusion and the balance equation of diffusion for the defect concentration. The developed model addresses the size effects through nonlocality. The defect concentration dynamics is governed by the generation and annihilation processes, as well as the strain-stimulated transport of defects due to nonlocal defectstrain interactions.

We have obtained dispersion equations for symmetric and antisymmetric modes in generalized forms through the solution of three coupled nonlocal equations, by assuming that the surface of the plates is stress-free. These equations contain complete information regarding wavenumber, plate thickness, phase velocity, group velocity, and attenuation (amplification) coefficients of different propagating modes. In first order approximation analytical expressions for determining the phase velocity and the attenuation (amplification) constants of the wave motion of the plate have been received. The formulae obtained in the paper, which are valid for nonlocal laser-excited elastic solid plates, may be used for the estimation of dispersion of general wave forms. From the results, it can be observed that the dispersion properties of the longitudinal waves are induced by the small scale effects and atomic defect generation, which will disappear in local continuous models and without defects. At short wavelength limits, the secular equations in the case of symmetric modes of wave propagation in the plate reduce to Rayleigh surface wave dispersion equations for half-spaces from [19]. Because in such situations the finite thickness layer appears as a halfspace and vibrational energy is transmitted mainly along the surface of the plate.

An approximate method of solution has been carried out for symmetric modes of wave propagation in the plate. This case shows appreciable difference from the local theory [22]. The same procedure can be applied to antisymmetric modes. Also, some special cases of the frequency equation have been discussed.

Clearly, the 2D linear theory considered here describes early stage of the development of the diffusion-elastic instability in isotropic solids only. However, the nature of generated ordered nanostructures (because of an instability) and the amplitudes of these structures as functions on material and irradiation conditions can only be determined by considering the influence of nonlinear effects in the model. In this regard, computer simulation of fluctuation development is needed. This nonlinear analysis will be considered in future paper. In addition, the combined effect of anisotropy and atomic defect generation on the wave propagation in laserexcited anisotropic semiconductor and dielectric crystalline solids or layers will be also investigated. Further research is necessary to understand the relations between the elastic and coupling coefficients of anisotropic materials and the characteristics of nonattenuating diffusion-elastic waves.

It is worth noting that the method developed in this paper can also be used to study the $3 \mathrm{D}$ problem of the wave 
propagation in an elastic layer. In this case the structure of resulting dispersion equation is the same as that obtained for plane waves in Section 5. For a semi-infinite isotropic elastic space, comparison of the dispersion equations obtained by use of $2 \mathrm{D}$ and $3 \mathrm{D}$ theories has been made in $[1,2]$.

The results obtained in this work are expected to be helpful in designing the various nanostructures in small scale devices.

\section{Conflict of Interests}

The author declares that there is no conflict of interests regarding the publication of this paper.

\section{Acknowledgment}

The author is grateful for useful comments of the reviewers.

\section{References}

[1] F. Kh. Mirzade, "On diffusion-elastic instabilities in a solid halfspace," Physica B: Condensed Matter, vol. 406, no. 1, pp. 119-124, 2011.

[2] F. Kh. Mirzade, "Concentration-elastic instabilities in a solid half-space," Physica Status Solidi (b), vol. 246, no. 7, pp. 15971603, 2009.

[3] F. Kh. Mirzade and V. Y. Panchenko, "Nonlinear strain waves interacting with laser-induced carries of the local disorder," in Laser Technologies of Materials Treatment, pp. 220-276, Fizmatlit, Moscow, Russia, 2009, (Russian).

[4] F. Kh. Mirzade, "A model for the propagation of strain solitary waves in solids with relaxing atomic defects," Journal of Applied Physics, vol. 103, Article ID 044904, 2008.

[5] D. Walgraef, N. M. Ghoniem, and J. Lauzeral, "Deformation patterns in thin films under uniform laser irradiation," Physical Review B, vol. 56, no. 23, pp. 15361-15377, 1997.

[6] D. Walgraef and N. M. Ghoniem, "Modeling laser-induced deformation patterns: nonlinear effects and numerical analysis," Journal of Computer-Aided Materials Design, vol. 6, no. 2, pp. 323-335, 1999.

[7] A. C. Eringen, Nonlocal Continuum Field Theories, Springer, New York, NY, USA, 2002.

[8] A. C. Eringen, "Theory of nonlocal elasticity and some applications," Res Mechanica, vol. 21, pp. 313-342, 1987.

[9] I. A. Kunin, Elastic Media with Microstructure II, Springer, New York, NY, USA, 1983.

[10] A. C. Eringen, "On differential equations of nonlocal elasticity and solutions of screw dislocation and surface waves," Journal of Applied Physics, vol. 54, no. 9, pp. 4703-4710, 1983.

[11] J. N. Reddy, "Nonlocal nonlinear formulations for bending of classical and shear deformation theories of beams and plates," International Journal of Engineering Science, vol. 48, no. 11, pp. 1507-1518, 2010.

[12] Y.-Z. Wang, F.-M. Li, and K. Kishimoto, "Scale effects on the longitudinal wave propagation in nanoplates," Physica E: LowDimensional Systems and Nanostructures, vol. 42, no. 5, pp. 1356-1360, 2010.

[13] M. Aydogdu, "Longitudinal wave propagation in nanorods using a general nonlocal unimodal rod theory and calibration of nonlocal parameter with lattice dynamics," International Journal of Engineering Science, vol. 56, pp. 17-28, 2012.
[14] P. Lu, P. Q. Zhang, H. P. Lee, C. M. Wang, and J. N. Reddy, "Nonlocal elastic plate theories," Proceedings of the Royal Society A: Mathematical, Physical \& Engineering Sciences, vol. 463, no. 2088, pp. 3225-3240, 2007.

[15] A. Alibeigloo, "Free vibration analysis of nano-plate using three-dimensional theory of elasticity," Acta Mechanica, vol. 222, no. 1-2, pp. 149-159, 2011.

[16] B. Arash and Q. Wang, "A review on the application of nonlocal elastic models in modeling of carbon nanotubes and graphenes," Computational Materials Science, vol. 51, no. 1, pp. 303-313, 2012.

[17] J. L. Nowiński, "On the nonlocal theory of wave propagation in elastic plates," ASME Journal of Applied Mechanics, vol. 51, no. 3, pp. 608-613, 1984.

[18] J. L. Nowiński, "On the propagation of thermoelastic waves in media with long-range cohesive forces," Journal of Thermal Stresses, vol. 10, no. 1, pp. 17-27, 1987.

[19] F. Kh. Mirzade, "Size effects on surface elastic waves in a semiinfinite medium with atomic defect generation," Advances in Condensed Matter Physics, vol. 2013, Article ID 528208, 11 pages, 2013.

[20] A. C. Eringen and D. G. Edelen, "On nonlocal elasticity," International Journal of Engineering Science, vol. 10, pp. 233-248, 1972.

[21] E. C. Aifantis and H. Askes, "Gradient elasticity and flexural wave dispersion in carbon nanotubes," Physical Review B, vol. 80, Article ID 195412, 2009.

[22] F. Kh. Mirzade, "Influence of atomic defect generation on the propagation of elastic waves in laser-excited solid layers," Physica B: Condensed Matter, vol. 406, no. 24, pp. 4644-4651, 2011.

[23] D. J. Achenbach, Wave Propagation in Elastic Solids, Elsevier, New York, NY, USA, 1973.

[24] F. Kh. Mirzade, "Influence of surface stress and atomic defect generation on Rayleigh wave propagation in laser-excited solids," Physica B: Condensed Matter, vol. 421, pp. 28-33, 2013.

[25] J. Gonzalo, A. Perea, D. Babonneau et al., "Competing processes during the production of metal nanoparticles by pulsed laser deposition," Physical Review B, vol. 71, no. 12, Article ID 125420, 2005. 

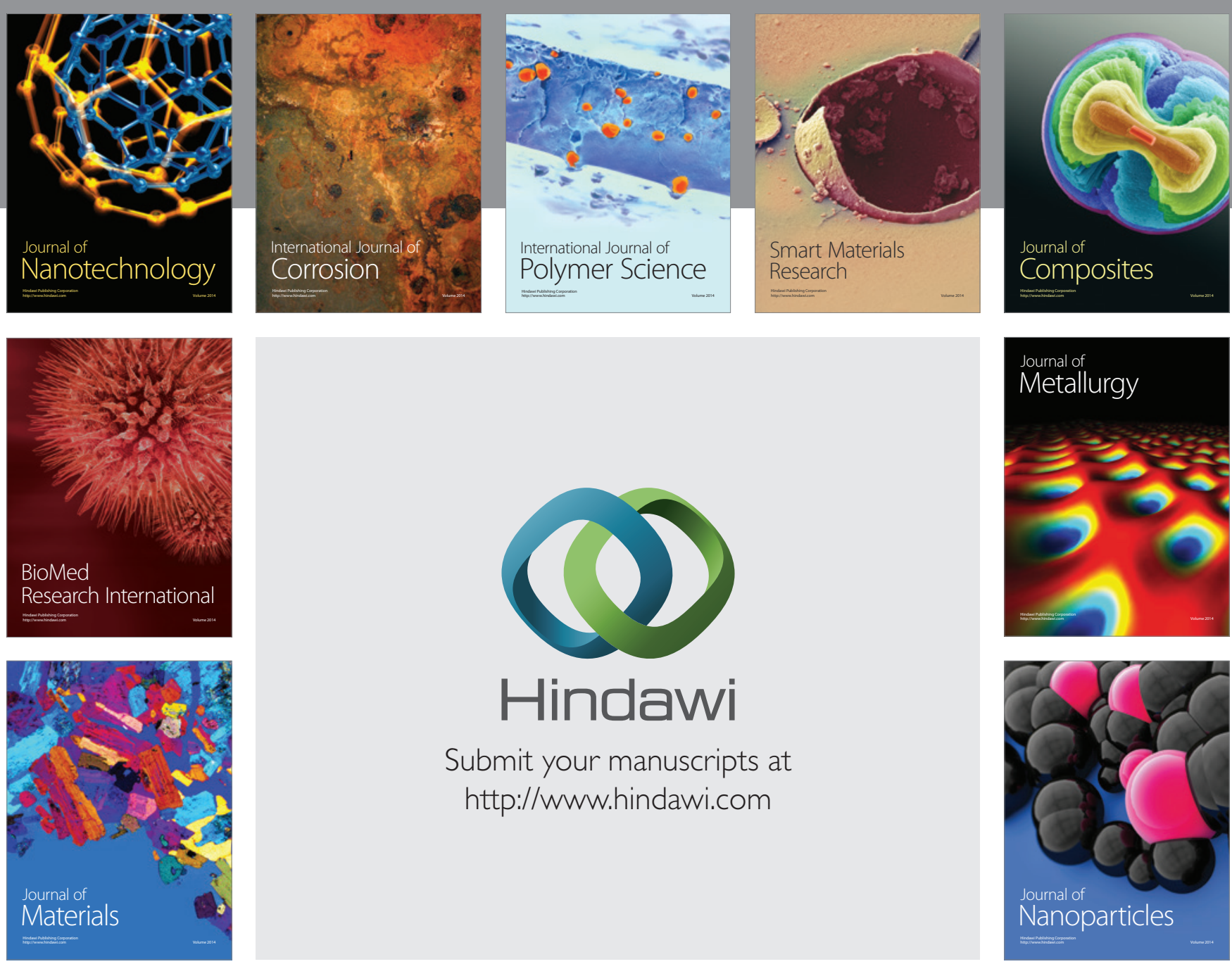

Submit your manuscripts at http://www.hindawi.com
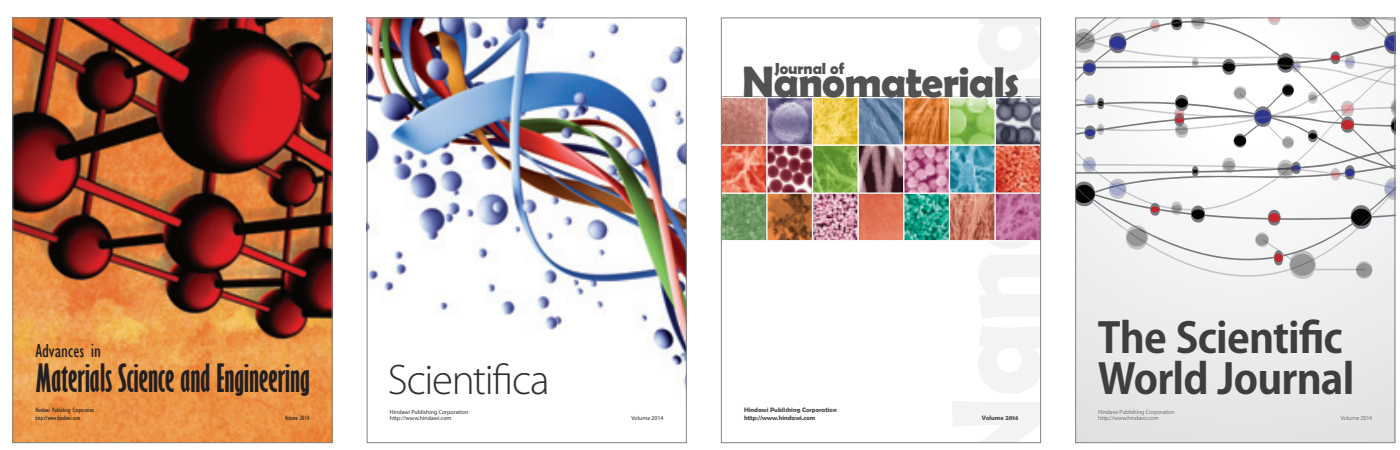

\section{The Scientific World Journal}
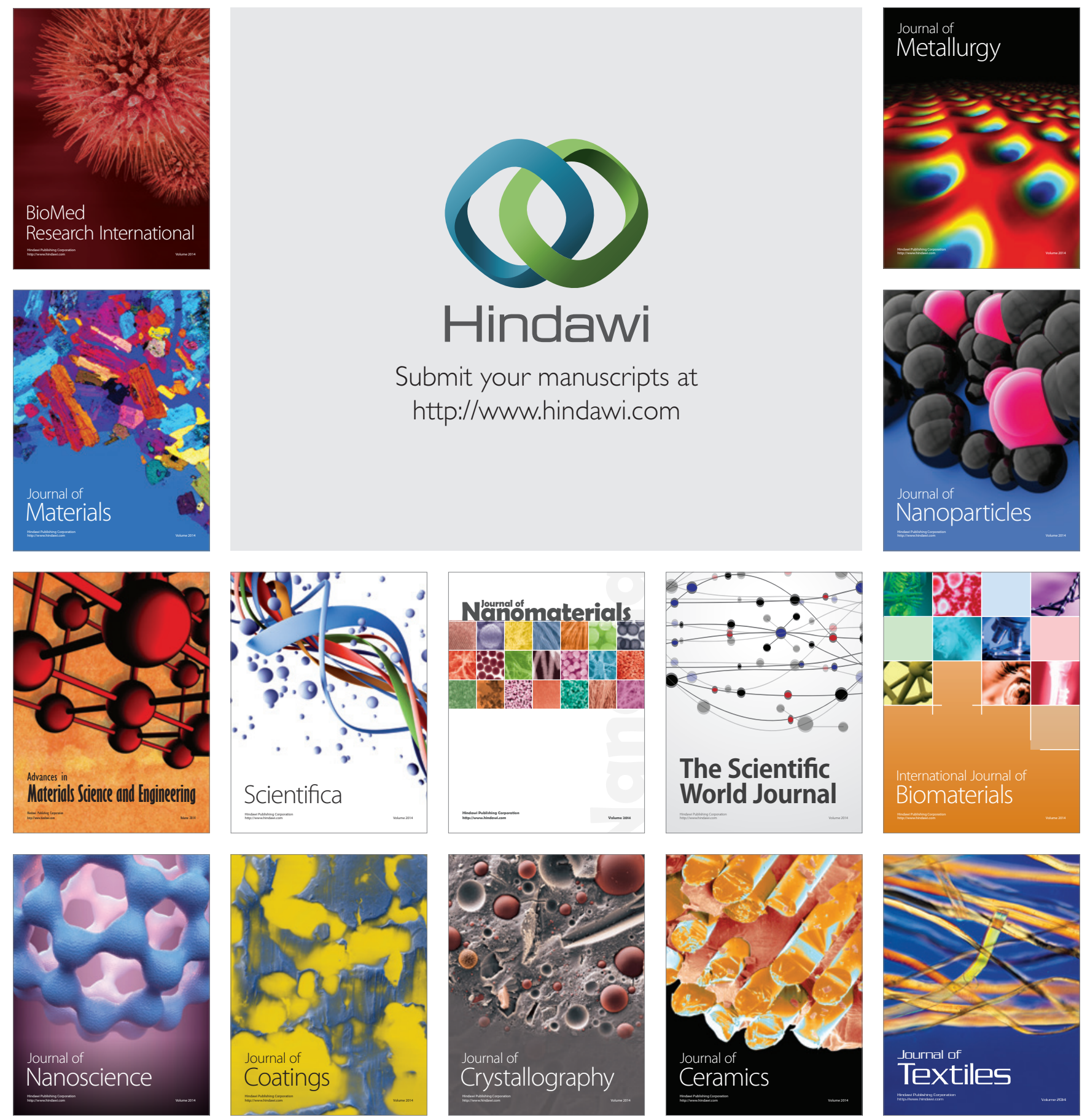